\title{
Efecto del ambiente rural sobre la prevalencia de rinitis alérgica en escolares de Mexicali, Baja California, México
}

\author{
Claudia Bäcker, ${ }^{1}$ Albino Barraza-Villarreal, ${ }^{1}$ Hortensia Moreno-Macías, ${ }^{1}$ \\ Consuelo Escamilla-Núñez ${ }^{1}$ e Isabelle Romieu ${ }^{1}$
}

Forma de citar $\quad \begin{aligned} & \text { Bäcker C, Barraza-Villarreal A, Moreno-Macías H, Escamilla-Núñez C, Romieu I. Efecto del ambiente } \\ & \text { rural sobre la prevalencia de rinitis alérgica en escolares de Mexicali, Baja California, México. Rev }\end{aligned}$ rural sobre la prevalencia de rinitis aler

RESUMEN Objetivos. Determinar la prevalencia de sintomas de rinitis alérgica en escolares de Mexicali, Baja California, México, y evaluar la influencia del medio ambiente rural sobre esta afección.

Métodos. Estudio transversal poblacional mediante la aplicación de un cuestionario estandarizado en una muestra aleatoria de 2087 escolares de 6 a 7 años (1 078 niñas y 1009 niños) residentes en la ciudad de Mexicali, Baja California, México. Se evaluó la asociación entre variables de exposición y antecedentes clinicos personales y familiares, y dos variables dependientes: sintomas nasales y sintomas nasales y oculares, en ausencia de catarro o gripe, con sus razones de posibilidades (OR, odds ratios) e intervalos de confianza de 95\% (IC95\%). El estudio, realizado entre febrero y julio de 2004, utilizó la metodología del Estudio Internacional de Asma y Alergia en la Infancia (ISAAC).

Resultados. Las prevalencias generales de sintomas nasales y sintomas nasales y oculares fueron de 25,0\% y 10,5\%, respectivamente. Los niños y las niñas que habían vivido en el campo o zona rural en algún momento presentaron menor probabilidad de padecer sintomas nasales $(O R=0,43$; IC95\%: 0,24 a 0,77) y sintomas nasales y oculares $(O R=0,39$; IC95\%: 0,16 a 0,93), después de ajustar por factores de confusión.

Conclusiones. El contacto con el medio rural en los primeros años de vida disminuyó el riesgo de presentar rinitis alérgica en la población estudiada, independientemente de los antecedentes familiares de asma.

Palabras clave Asma, rinitis alérgica estacional, rinitis alérgica perenne, niño, medio ambiente y salud pública, México.

La incidencia de enfermedades atópicas, como el asma, la rinitis alérgica y el eczema, se ha incrementado en la pobla-

\footnotetext{
Instituto Nacional de Salud Pública, Cuernavaca, Morelos, México. La correspondencia se debe dirigir a Isabelle Romieu, Instituto Nacional de Salud Pública, Av. Universidad No. 655, Col. Santa María Ahuacatitlán, Cuernavaca, Morelos 62100, México. Correo electrónico: iromieu@correo. insp.mx
}

ción infantil en las últimas décadas $(1,2)$. Existen múltiples teorías que intentan explicar las razones de este incremento, entre las que se destacan las basadas en los factores genéticos (3), el estilo de vida y las condiciones del medio ambiente (4). Una de las teorías más relevantes es la llamada hipótesis higiénica, que postula que una forma natural de modular la respuesta inmunitaria es entrar en con- tacto con alérgenos y microorganismos (o partes de ellos) en etapas tempranas de la vida (5-7). En ese sentido, se ha comprobado que el ambiente rural o campestre permite tener un mayor contacto con animales y microorganismos y permite llevar un estilo de vida con las características peculiares de ese entorno que favorecen el proceso de modulación inmunitaria $(8,9)$. 
Por otro lado, se sabe que un limitado grado de exposición a bacterias y virus puede producir un estímulo insuficiente de linfocitos Th1, lo que llevaría a un mayor desarrollo de los linfocitos Th2, asociados con procesos alérgicos (7).

En los últimos años, numerosos autores han observado que la prevalencia de enfermedades atópicas en la infancia es menor en niños y niñas que viven en zonas rurales en comparación con los que viven en zonas urbanas (10-12); sin embargo, el tema se mantiene controversial, ya que otros autores han encontrado resultados diferentes $(13,14)$. El objetivo del presente trabajo fue determinar la prevalencia de síntomas de rinitis alérgica en escolares de Mexicali, Baja California, México, y evaluar la influencia del medio ambiente rural sobre esta afección.

\section{MATERIALES Y MÉTODOS}

Se realizó un estudio transversal poblacional mediante la aplicación de un cuestionario estandarizado a una muestra aleatoria de 2087 escolares de 6 a 7 años de edad residentes en la ciudad de Mexicali, capital del estado de Baja California, al norte de México. El estudio utilizó la metodología del Estudio Internacional de Asma y Alergia en la Infancia (ISAAC) (15).

El universo de estudio estuvo constituido por todas las escuelas de educación básica, tanto públicas como privadas, de Mexicali, según el padrón provisto por la Secretaría de Educación Pública de Baja California. Se seleccionaron 55 escuelas de manera aleatoria. Según lo recomendado por el Estudio ISAAC, el tamaño escogido para la muestra fue de 3000 niños, más $20 \%$ adicional para cubrir posibles pérdidas. Se invitó a participar a todos los alumnos de 6 y 7 años de edad inscritos en el primer grado de educación básica de las escuelas seleccionadas.

La evaluación de los síntomas de rinitis alérgica se llevó a cabo entre febrero y julio de 2004 mediante un cuestionario validado y estandarizado por ISAAC (16), que ya se ha utilizado exitosamente en varios países, entre ellos México (17, 18). Este cuestionario contiene preguntas sobre la presencia de síntomas y diagnósticos médicos de asma, rinitis alérgica y eczema; características sociodemográficas del niño y su familia; condiciones de la vivienda y cercanía al campo o zonas rurales; exposición a la humedad, contaminantes, tabaquismo pasivo y plaguici- das en el interior de la vivienda; antecedentes médicos del niño, en particular de infecciones respiratorias que hayan requerido hospitalización en sus primeros años de vida; y antecedentes médicos de sus familiares, en particular de asma y alergias. Los síntomas de rinitis alérgica se abordaron mediante dos variables dependientes: síntomas nasales (presencia de estornudos, obstrucción nasal o mucosidad en ausencia de catarro o gripe, en los 12 meses previos a la entrevista) y síntomas nasales y oculares (presencia de estornudos, obstrucción nasal o mucosidad acompañados de lagrimeo y comezón en los ojos, en ausencia de catarro o gripe, en los 12 meses previos a la entrevista).

En el cuestionario se preguntó también si al nacimiento o en algún momento, el niño había vivido en el campo o zona rural, así como la edad que tenía el niño y la duración de esa estancia. También se abordó el contacto del niño con animales durante sus primeros años de vida y en el momento de la entrevista. Los padres o tutores de los menores respondieron los cuestionarios de manera individual (autoadministrado) una vez que dieron su consentimiento por escrito.

Esta investigación contó con la aprobación del Comité de Ética del Instituto Nacional de Salud Pública de México.

\section{Análisis estadístico}

Se realizó un análisis exploratorio de la base de datos con el fin de detectar posibles errores y caracterizar el grupo de estudio mediante medidas de frecuencia y de tendencia central (media y desviación estándar). Posteriormente se efectuó un análisis entre pares de variables para evaluar la asociación entre las variables dependientes y las variables de exposición, de confusión y demás variables estudiadas. De estas variables probadas, las que mostraron una asociación suficiente $(P<0,10)$ y biológicamente plausible con las variables dependientes se utilizaron en los modelos de regresión logística múltiple a partir de un modelo saturado y las variables que tuvieron una asociación estadística significativa $(P<0,05)$ en la regresión múltiple y que eran biológicamente plausibles se dejaron en el modelo final. Como factores de confusión para ajustar los modelos se emplearon el tipo de escuela, la exposición a plaguicidas en el interior de la vivienda, la humedad en la vi- vienda, la exposición al tabaquismo pasivo y los antecedentes de asma o rinitis alérgica. Se calcularon las razones de posibilidades (odds ratio, OR) con sus correspondientes intervalos de confianza de $95 \%$ (IC95\%) y se exploró la posible modificación del efecto (interacción) de las variables empleadas. Para el análisis estadístico se utilizó el paquete estadístico STATA, versión 8.0 (19).

\section{RESULTADOS}

De un total de 3125 niños y niñas elegibles, completaron los cuestionarios 2528 , para una tasa de respuesta de $81,2 \%$. No obstante, para el análisis solamente se tomaron 2087 cuestionarios, correspondientes a los niños de 6-7 años (1 078 niñas y 1009 niños) (cuadro 1). Las proporciones de niños $(49,9 \%)$ y niñas $(50,1 \%)$ de 6 y 7 años fueron muy similares $(P=0,528)$.

Hubo una mayor proporción de escuelas públicas $(82,6 \%)$ que privadas $(17,4 \%)$, localizadas en su mayoría en áreas urbanas $(94,2 \%)$. En lo referente al nivel escolar cumplido por la madre, la mayoría había terminado la educación primaria o secundaria $(61,2 \%)$; la mayor parte de los participantes $(80,3 \%)$ tenía al menos un hermano mayor (cuadro 1).

La prevalencia general de síntomas nasales fue de $25,0 \%$, menor en niñas $(21,1 \%)$ que en niños $(29,1 \% ; P<0,01)$, mientras que la prevalencia de síntomas nasales y oculares fue de $10,5 \%$ sin diferencias entre niños y niñas (figura 1).

En el análisis de pares de variables se observó que vivir en el campo o zona rural en algún momento de la vida funcionó como un factor de protección contra los síntomas nasales ( $\mathrm{OR}=0,63$; IC95\%: 0,41 a 0,96), al igual que haber estado en contacto con animales durante el tiempo que habitó en el campo o zona rural (OR =0,48; IC95\%: 0,27 a 0,84). Sin embargo, el decremento observado en el riesgo de tener síntomas nasales y oculares en los niños y niñas que vivieron en el campo o zona rural en algún momento no fue estadísticamente significativo $(\mathrm{OR}=0,59$; IC95\%: 0,30 a 1,13) (cuadro 2). No se observaron asociaciones significativas en relación con la edad a la cual los participantes vivieron en el campo o zona rural. Sin embargo, el efecto protector para los síntomas nasales se incrementó conforme aumentaron los años de vida en el campo o zona rural (prueba de tendencia $P=0,04)$. 
CUADRO 1. Características de la población estudiada de escolares de Mexicali, Baja California, México, febrero-julio de 2004

\begin{tabular}{lrr}
\hline \multicolumn{1}{c}{ Variable $^{\mathrm{a}}$} & No. & $\%$ \\
\hline Sexo $(n=2$ 087) & & \\
$\quad$ Niñas & 1078 & 51,7 \\
$\quad$ Niños & 1009 & 48,3 \\
Nivel escolar (terminado) de la madre $(n=2$ 061) & 40 & 2,0 \\
$\quad$ Sin escolaridad & 1262 & 61,2 \\
$\quad$ Primaria o secundaria & 759 & 36,8 \\
$\quad$ Preparatoria o licenciatura & & \\
Número de hermanos mayores $(n=1507)$ & 297 & 19,7 \\
$\quad$ Ninguno & 654 & 43,4 \\
Uno & 556 & 36,9 \\
$\quad$ Dos o más & & \\
Tipo de escuela ( $n=2$ 067) & 360 & 17,4 \\
$\quad$ Privada & 1707 & 82,6 \\
$\quad$ Pública & & \\
Ubicación de la escuela $(n=2$ 067) & 119 & 5,8 \\
$\quad$ Rural & 1948 & 94,2 \\
Urbana & & \\
\hline
\end{tabular}

${ }^{a}$ El número de respuestas varía debido a datos faltantes.
Con respecto a las otras variables evaluadas, se observó que tener familiares con antecedentes de asma o rinitis alérgica fue un factor de riesgo significativo tanto para los síntomas nasales (OR = 3,42; IC95\%: 2,69 a 4,35) como para los nasales y oculares $(\mathrm{OR}=3,33$; IC95\%: 2,44 a 4,56), mientras que ser del sexo masculino (OR = 1,53; IC95\%: 1,24 a $1,88)$ solo fue estadísticamente significativo para los síntomas nasales. Por otro lado, el tener dos hermanos o más fue un factor de protección (OR =0,66; IC95\%: 0,46 a 0,92 ) para los síntomas nasales

Con respecto a las características de la vivienda, los factores de riesgo tanto para los síntomas nasales como para los sínto(cuadro 2). mas nasales y oculares fueron: la presencia de humedad (OR = 2,18; IC95\%: 1,58 a 3,02 y OR = 2,48; IC95\%: 1,65 a 3,75, respectivamente), la exposición a plaguicidas en el interior de la vivienda $(\mathrm{OR}=2,16 ;$ IC95\%: 1,64 a 2,85 y $\mathrm{OR}=2,12$; IC95\%: 1,42 a 3,17, respectivamente) y la ubicación de la vivienda actual cerca de algún campo o zona rural $(\mathrm{OR}=1,62$; IC95\%: 1,17 a 2,24 y OR = 1,63; IC95\%: 1,06 a 2,50, respectivamente). El asistir a una escuela pública $(\mathrm{OR}=0,55$; IC95\%: 0,43 a 0,70) fue un factor de protección con respecto a asistir a una escuela privada $(\mathrm{OR}=0,44$; IC95\%: 0,32 a 0,61$)$ para ambos síntomas (cuadro 2).

$\mathrm{Al}$ incorporar al análisis las variables de exposición (vivir en el campo o zona
FIGURA 1. Prevalencia de síntomas de rinitis alérgica en los 12 meses previos al estudio en escolares de Mexicali, Baja California, México, febrero-julio de 2004

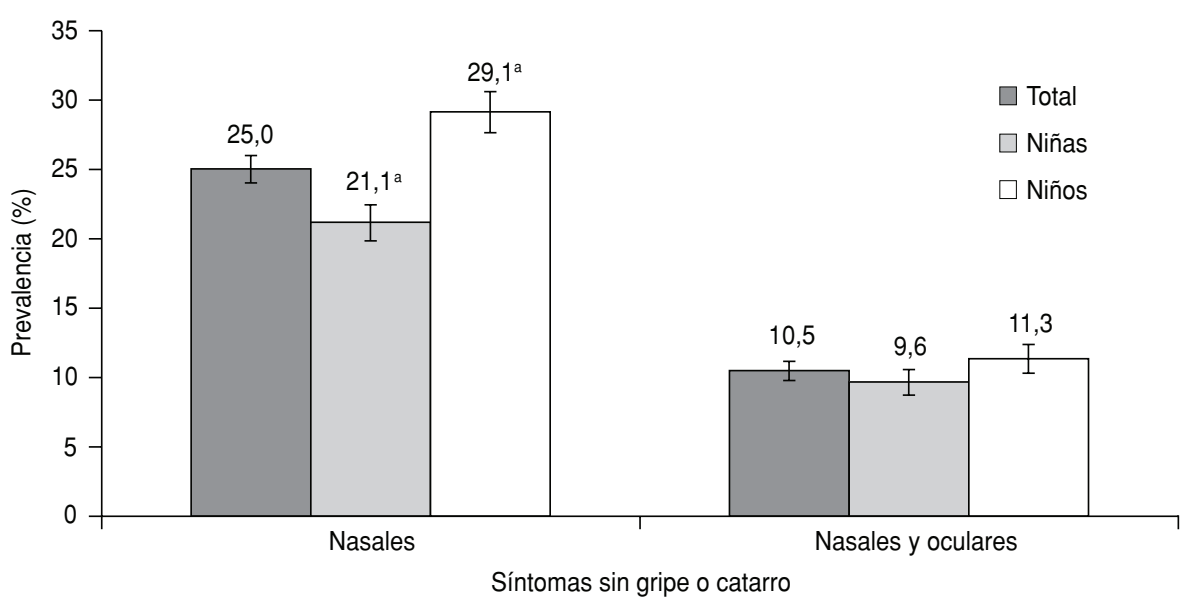

${ }^{a}$ Diferencia significativa entre las prevalencias en niñas y niños $(P<0,05)$. rural y tener contacto con animales en el momento del estudio) y de confusión (tipo de escuela, presencia de humedad en la vivienda, uso de plaguicidas en el interior de la vivienda, exposición al humo de tabaco en el momento del estudio y antecedentes familiares de asma o rinitis alérgica) simultáneamente, el haber vivido en el campo o zona rural en algún momento se mantuvo como un factor de protección significativo tanto para los síntomas nasales (OR $=0,43$; IC95\%: $0,24$ a 0,77$)$ como para los nasales y oculares $(\mathrm{OR}=0,39$; IC95\%: 0,16 a 0,93).

Cuando se estratificó el análisis de acuerdo con los antecedentes familiares de asma o rinitis alérgica, se encontró que haber vivido en el campo o zona rural se mantuvo como factor protector significativo para los síntomas nasales en los niños y las niñas con antecedentes familiares de asma o rinitis alérgica $(\mathrm{OR}=0,32$; IC95\%: 0,13 a 0,79) (cuadro 3). En cuanto al contacto con animales en el momento del estudio, también se observó una disminución en la frecuencia de síntomas nasales y oculares en todos los niños y niñas estudiados $(\mathrm{OR}=0,78$; IC95\%: 0,59 a 1,03 y OR =0,65; IC95\%: 0,45 a 0,95 , respectivamente). Sin embargo, después de la estratificación por los antecedentes familiares de asma o rinitis alérgica, el contacto con animales en el momento del estudio se mantuvo significativo solo en los niños y las niñas sin antecedentes familiares de asma o rinitis alérgica para ambos síntomas de rinitis alérgica (OR =0,63; IC95\%: 0,46 a 0,88 y $\mathrm{OR}=0,54$; IC $95 \%: 0,34$ a 0,87 , respectivamente) (cuadro 3).

\section{DISCUSIÓN}

En este estudio transversal poblacional basado en la metodología ISAAC se encontró que vivir por algún tiempo en el campo o zona rural a edades más tempranas y el estar en contacto con animales durante el momento de la realización del estudio disminuyó la frecuencia de síntomas nasales y los síntomas nasales y oculares en escolares de la ciudad de Mexicali.

Con anterioridad, la metodología ISAAC se ha empleado en estudios realizados tanto en México - Cuernavaca (20), Chihuahua $(21,22)$ y el Distrito Federal (23) - como en otros países de América Latina (24) y otras regiones (25). Sin embargo, esta es la primera vez que se emplea esta metodología para evaluar 
CUADRO 2. Asociación entre los síntomas de rinitis alérgica en los 12 meses previos a la encuesta y las principales variables estudiadas, según el análisis con dos variables, en escolares de Mexicali, Baja California, México, febrero-julio de 2004

\begin{tabular}{|c|c|c|c|c|c|c|c|}
\hline \multirow[b]{2}{*}{ Variable } & \multirow[b]{2}{*}{ No. } & \multicolumn{3}{|c|}{ Síntomas nasales ${ }^{a}$} & \multicolumn{3}{|c|}{ Síntomas nasales y oculares ${ }^{a}$} \\
\hline & & No. & $\mathrm{OR}^{\mathrm{b}}$ & IC95\% ${ }^{\mathrm{C}}$ & No. & OR & IC95\% \\
\hline \multicolumn{8}{|c|}{$\begin{array}{l}\text { Vivir en el campo o zona rural antes del primer } \\
\text { año de vida }(n=1932)\end{array}$} \\
\hline Nod & 1820 & 422 & 1,0 & & 173 & 1,0 & \\
\hline Sí & 112 & 20 & 0,76 & $0,46-1,26$ & 8 & 0,76 & $0,36-1,58$ \\
\hline \multicolumn{8}{|c|}{$\begin{array}{l}\text { Vivir en algún momento en el campo o zona rural } \\
(n=1931)\end{array}$} \\
\hline $\mathrm{No}^{\mathrm{d}}$ & 1756 & 405 & 1,0 & & 171 & 1,0 & \\
\hline Sí & 175 & 27 & 0,63 & $0,41-0,96^{\mathrm{e}}$ & 10 & 0,59 & $0,30-1,13$ \\
\hline \multicolumn{8}{|c|}{$\begin{array}{l}\text { Edad a la que vivió en el campo o zona rural } \\
\text { (años) }(n=1928)\end{array}$} \\
\hline Nunca $^{d}$ & 1756 & 405 & 1,0 & & 171 & 1,0 & \\
\hline $0-2$ & 126 & 25 & 0,84 & $0,53-1,32$ & 10 & 0,83 & $0,42-1,61$ \\
\hline $2-5$ & 46 & 6 & 0,56 & $0,23-1,35$ & 2 & 0,47 & $0,11-1,96$ \\
\hline \multicolumn{8}{|c|}{$\begin{array}{l}\text { Tiempo que vivió en el campo o zona rural } \\
\text { (años) }(n=1923)\end{array}$} \\
\hline Nunca ${ }^{d}$ & 1756 & 405 & 1,0 & & 171 & 1,0 & \\
\hline$<1$ & 78 & 14 & 0,78 & $0,43-1,43^{\dagger}$ & 3 & 0,41 & $0,13-1,31$ \\
\hline $1-5$ & 74 & 13 & 0,64 & $0,34-1,20^{f}$ & 4 & 0,53 & $0,19-1,46$ \\
\hline$>5$ & 15 & 2 & 0,26 & $0,03-1,98^{\dagger}$ & 1 & 0,72 & $0,09-5,59$ \\
\hline \multicolumn{8}{|c|}{ Contacto con animales al vivir en el campo o } \\
\hline Nod & 1814 & 420 & 1,0 & & 177 & 1,0 & \\
\hline Sí & 120 & 15 & 0,48 & $0,27-0,84 \mathrm{e}$ & 7 & 0,6 & $0,27-1,31$ \\
\hline \multicolumn{8}{|l|}{$\operatorname{Sexo}(n=2087)$} \\
\hline Niña ${ }^{d}$ & 1078 & 212 & 1,0 & & 97 & 1,0 & \\
\hline Niño & 1009 & 274 & 1,53 & $1,24-1,88^{e}$ & 106 & 1,2 & $0,89-1,60$ \\
\hline \multicolumn{8}{|c|}{$\begin{array}{l}\text { Antecedentes familiares de asma o rinitis alérgica } \\
(n=1827)\end{array}$} \\
\hline $\mathrm{No}^{\mathrm{d}}$ & 1412 & 320 & 1,0 & & 139 & 1,0 & \\
\hline Sí & 415 & 112 & 3,42 & $2,69-4,35^{\mathrm{e}}$ & 45 & 3,33 & $2,44-4,56^{e}$ \\
\hline \multicolumn{8}{|c|}{ Exposición al humo de tabaco en la vivienda ( $n=1945$ ) } \\
\hline Nod & 1561 & 357 & 1,0 & & 155 & 1,0 & \\
\hline Sí & 384 & 102 & 1,25 & $0,96-1,62$ & 41 & 1,1 & $0,77-1,6$ \\
\hline \multicolumn{8}{|c|}{ Presencia de humedad en la vivienda ( $n=1932$ ) } \\
\hline Nod & 1736 & 376 & 1,0 & & 152 & 1,0 & \\
\hline Sí & 196 & 69 & 2,18 & $1,58-3,02^{\mathrm{e}}$ & 34 & 2,48 & $1,65-3,75^{\mathrm{e}}$ \\
\hline \multicolumn{8}{|l|}{$\begin{array}{l}\text { Exposición a plaguicidas en el } \\
\text { interior de la vivienda }(n=1757)\end{array}$} \\
\hline Nod & 482 & 75 & 1,0 & & 31 & 1,0 & \\
\hline Sí & 1275 & 351 & 2,16 & $1,64-2,85^{\mathrm{e}}$ & 158 & 2,12 & $1,42-3,17^{e}$ \\
\hline \multicolumn{8}{|c|}{ Vivir cerca del campo o zona rural $(n=1950)$} \\
\hline Nod & 1748 & 392 & 1,0 & & 167 & 1,0 & \\
\hline Sí & 202 & 62 & 1,62 & $1,17-2,24^{e}$ & 29 & 1,63 & $1,06-2,50^{e}$ \\
\hline \multicolumn{8}{|c|}{$\begin{array}{l}\text { Contacto con animales en el momento del estudio } \\
(n=2044)\end{array}$} \\
\hline Nod & 831 & 202 & 1,0 & & 90 & 1,0 & \\
\hline Sí & 1213 & 279 & 0,96 & $0,78-1,19$ & 113 & 0,88 & $0,65-1,17$ \\
\hline \multicolumn{8}{|c|}{ Nivel escolar (cumplido) de la madre $(n=2061)$} \\
\hline Sin escolaridad ${ }^{d}$ & 40 & 8 & 1,0 & & 2 & 1,0 & \\
\hline Primaria o secundaria & 1262 & 251 & 1,04 & $0,47-2,30$ & 95 & 1,61 & $0,38-6,79$ \\
\hline Preparatoria o licenciatura & 759 & 223 & 1,66 & $0,75-3,68$ & 108 & 3,16 & $0,75-13,34$ \\
\hline \multicolumn{8}{|c|}{ Número de hermanos mayores $(n=1507)$} \\
\hline Ningunod & 297 & 76 & 1,0 & & 34 & 1,0 & \\
\hline Uno & 654 & 177 & 1,11 & $0,81-1,52$ & 80 & 1,1 & $0,72-1,69$ \\
\hline Dos o más & 556 & 97 & 0,66 & $0,46-0,92^{\mathrm{e}}$ & 46 & 0,74 & $0,46-1,18$ \\
\hline Tipo de escuela $(n=2$ 067) & & & & & & & \\
\hline Privada $^{d}$ & 360 & 268 & 1,0 & & 112 & 1,0 & \\
\hline Pública & 1707 & 119 & 0,55 & $0,43-0,70^{e}$ & 92 & 0,44 & $0,32-0,61^{e}$ \\
\hline Ubicación de la escuela ( $n=2$ & & & & & & & \\
\hline Rurald $^{d}$ & 119 & 170 & 1,0 & & 72 & 1,0 & \\
\hline Urbana & 1948 & 318 & 0,97 & $0,63-1,51$ & 133 & 1,08 & $0,57-2,05$ \\
\hline
\end{tabular}


CUADRO 3. Asociación entre los síntomas de rinitis alérgica en los 12 meses previos a la entrevista, según el análisis con múltiples variables estratificado por los antecedentes familiares de asma o rinitis alérgica, en escolares de Mexicali, Baja California, México, febrero-julio de 2004

\begin{tabular}{|c|c|c|c|c|c|c|}
\hline \multirow[b]{2}{*}{ Modelo/variable } & \multicolumn{3}{|c|}{ Síntomas nasales } & \multicolumn{3}{|c|}{ Síntomas nasales y oculares } \\
\hline & No. ${ }^{a}$ & $\mathrm{OR}^{\mathrm{b}}$ & IC95\% ${ }^{\mathrm{C}}$ & No. ${ }^{a}$ & OR & IC95\% \\
\hline \multicolumn{7}{|l|}{ Generald $^{d}$} \\
\hline Vivir en algún momento en el campo o zona rural & 27 & 0,43 & $0,24-0,77^{e}$ & 10 & 0,39 & $0,16-0,93^{e}$ \\
\hline Contacto con animales en el momento del estudio & 279 & 0,78 & $0,59-1,03^{f}$ & 113 & 0,65 & $0,45-0,95^{\oplus}$ \\
\hline Total en el modelo ajustado & 1237 & & & 1236 & & \\
\hline \multicolumn{7}{|l|}{ Con antecedentes familiares de asma o rinitis alérgicag } \\
\hline Vivir en algún momento en el campo o zona rural & 8 & 0,32 & $0,13-0,79 e$ & 4 & 0,60 & $0,22-1,66$ \\
\hline Contacto con animales en el momento del estudio & 65 & 1,32 & $0,77-2,25$ & 22 & 0,86 & $0,47-1,57$ \\
\hline Total en el modelo ajustado & 276 & & & 274 & & \\
\hline \multicolumn{7}{|l|}{ Sin antecedentes familiares de asma o rinitis alérgica ${ }^{g}$} \\
\hline Vivir en algún momento en el campo o zona rural & 17 & 0,54 & $0,26-1,14$ & 4 & 0,14 & $0,02-1,03^{\dagger}$ \\
\hline Contacto con animales en el momento del estudio & 179 & 0,63 & $0,46-0,88^{\mathrm{e}}$ & 77 & 0,54 & $0,34-0,87^{e}$ \\
\hline Total en el modelo ajustado & 961 & & & 962 & & \\
\hline
\end{tabular}

a El número de respuestas para cada variable varió debido a datos faltantes.

b OR: razón de posibilidades (odds ratio).

c IC95\%: intervalo de confianza de $95 \%$.

d Modelo ajustado por el tipo de escuela, la exposición a plaguicidas, la humedad en la vivienda, la exposición al tabaquismo pasivo y los antecedentes de asma o rinitis alérgica.

e Diferencia significativa $(P<0,05)$

f Sin diferencia significativa $(0,05<P<0,10)$, pero incorporado a los modelos con múltiples variables (ajustados).

g Modelo ajustado por el tipo de escuela, la exposición a plaguicidas, la humedad en la vivienda y la exposición al tabaquismo pasivo.

el efecto del ambiente rural en los síntomas de rinitis alérgica en México.

Según los resultados del presente estudio, las prevalencias generales tanto de los síntomas nasales $(25,0 \%)$ como de los nasales y oculares $(10,5 \%)$ en los niños y las niñas de 6-7 años estudiados son cercanas a las encontradas en otras ciudades de México, tanto para los síntomas nasales $(24,8 \%$ a $28,8 \%)$ como para los nasales y oculares $(9,6 \%$ a $17,0 \%)$ en este grupo de edad (20-22). No obstante, las prevalencias encontradas aquí difieren de las informadas en otros países de América Latina (24), lo cual puede ser el reflejo de diferencias en las poblaciones estudiadas, sus estilos de vida o las condiciones del medio ambiente de sus respectivas localidades. Los niños que habían vivido en el campo o zona rural durante los primeros años de vida presentaron una menor prevalencia de síntomas nasales que sus pares que no tuvieron esa experiencia, y sus probabilidades de presentar síntomas nasales o síntomas nasales y oculares también fueron menores. Estos resultados son consistentes con informes de otros países $(9,10,26,27)$.

El mecanismo propuesto para explicar por qué el ambiente rural brinda una mayor protección contra el desarrollo de enfermedades atópicas en los niños y sus manifestaciones graves (hospitalización por asma) se basa en que la vida en el campo o zona rural favorece un estilo de vida más "tradicional", con familias más numerosas, una dieta más natural y sin la exposición a los contaminantes ambientales propios de las grandes urbes (12). Se considera que el contacto con los animales domésticos (perros y gatos) y de corral (cerdos, vacas y caballos, entre otros) en los primeros años de vida puede favorecer el desarrollo temprano del sistema inmunitario de los niños al entrar en contacto con un espectro más amplio de antígenos. Asimismo se ha observado que el contacto con diferentes agentes bacterianos no infecciosos y endotoxinas en ese ambiente, también puede influir positivamente en la modulación de la respuesta inmunitaria de los niños (2). La concentración de endotoxinas es elevada en las granjas y el polvo doméstico, pero es mucho mayor en establos y corrales, y se ha encontrado una correlación inversa entre el nivel de exposición a endotoxinas y la probabilidad de los niños de padecer rinitis alérgica, asma y sensibilización atópica (26-28).

Otro elemento importante es la edad y el tiempo de residencia en el campo o zona rural, ya que la protección contra la rinitis alérgica es mayor cuanto más temprana es esa exposición. Esto puede deberse a que en los primeros años de vida, el sistema inmunitario es más susceptible al proceso de modulación (11). Sin embargo, en este estudio no se encontró una diferencia marcada entre el efecto protector en los niños que permanecie- ron en el campo o zona rural antes de cumplir 2 años y los que permanecieron allí entre los 2 y los 5 años.

Por otro lado, se sabe que en los recién nacidos predominan los linfocitos Th2 del sistema inmunitario fetal y posteriormente se van reemplazando por los linfocitos Th1. Si el niño entra en contacto con antígenos a más temprana edad se favorece el desplazamiento de la respuesta inmunitaria hacia los linfocitos Th1 (mediante el interferón $\gamma$ y la interleucina 2) más que hacía los linfocitos Th2 (por las interleucinas 4 y 5) y se regula la producción de la inmunoglobulina E en los linfocitos B $(26,28)$. Según diversos autores, el desplazamiento hacia la respuesta inmunitaria basada en los linfocitos Th1 se manifestará más tarde en una menor prevalencia de enfermedades atópicas (29-32).

En este estudio, el efecto protector aumentó a medida que fue mayor el tiempo de permanencia del niño o la niña en el campo o zona rural. Esto respalda la afirmación de otros autores que encontraron que a mayor tiempo de permanencia en el campo o zona rural (medido indirectamente por las tareas efectuadas por las familias en el campo o zona rural a tiempo completo o parcial, y el tiempo de visita o permanencia del niño o la niña en el establo), mayor fue el efecto protector de esa exposición sobre el riesgo de presentar sibilancia o rinitis alérgica (11). 
Otro hallazgo importante se observó al estratificar el análisis por los antecedentes familiares de asma o rinitis alérgica, que mostró que el efecto protector del ambiente campestre o rural con respecto a los síntomas de rinitis alérgica (síntomas nasales y síntomas nasales y oculares) se mantuvo, independientemente de los otros factores de riesgo implicados en el desarrollo de estos síntomas, incluso en niños con esos antecedentes. Esto confirma que si bien la intervención es difícil cuando hay una carga genética, sí se puede reducir el riesgo de rinitis alérgica al mejorar los factores ambientales gracias a su influencia en el desarrollo de los síntomas estudiados.

Asimismo, durante la estratificación se observó una frecuencia menor en los síntomas cuando se informó que el niño estaba en contacto con animales en el momento del estudio, pero este posible efecto protector resultó significativo solamente en los niños que no tenían antecedentes familiares de asma o rinitis alérgica. Esto podría indicar que el contacto más tardío con animales no influye significativamente en la predisposición genética de la rinitis alérgica. Resultados similares se han encontrado en estudios prospectivos que evaluaron la exposición temprana a perros en meno- res sin antecedentes familiares de asma (33).

En cuanto a la evaluación de otros factores directa o indirectamente relacionados con la rinitis alérgica, se encontró que el asistir a escuelas públicas redujo el riesgo de los síntomas estudiados (20). Este resultado respalda resultados anteriores en el sentido de que el contacto más temprano e intenso con otros niños (lo que implica tener más riesgo de padecer infecciones a menor edad) desempeña un papel protector contra la aparición de enfermedades alérgicas en etapas posteriores de la vida (6), sin embargo aún hay controversia al respecto ya que otros estudios han encontrado un aumento en el riesgo (21).

Por último, es importante tener en cuenta algunas limitaciones al interpretar estos resultados. El diseño transversal de este estudio no permite evaluar la temporalidad de los fenómenos estudiados. Debido a que las conclusiones se basan en las respuestas dadas a una encuesta, existe la posibilidad de que haya habido un sesgo de memoria, pues la exposición se evaluó de manera retrospectiva para los primeros años de vida de los niños; esto puede ocasionar una respuesta diferencial, especialmente en los padres de los niños y niñas que padecían rinitis alérgica, ya que podrían estar más condicionados a recordar ciertas exposiciones. Por otra parte, es posible que los padres hayan reducido intencionalmente el contacto de sus hijos alérgicos con los animales, por saber que esto podía desencadenar o agravar las reacciones alérgicas del niño. Sin embargo, dado que los niños y los padres participantes no conocían la hipótesis del estudio y posiblemente no tenían suficiente conocimiento acerca de la posible influencia de vivir o haber vivido en el campo o zonas rurales, es poco probable que esto haya afectado a los resultados. Asimismo, es importante resaltar que el instrumento utilizado para evaluar los síntomas estudiados es un cuestionario bien validado, lo que reduce la posibilidad de un error de medición (34).

En conclusión, en este estudio se comprobó el efecto protector del ambiente rural o campestre sobre los síntomas de rinitis alérgica en una población escolar de la ciudad de Mexicali, México. Esta asociación es independiente de los antecedentes familiares de asma. Se recomienda realizar estudios prospectivos que evalúen la influencia del ambiente rural y determinen el papel que desempeña esta exposición en la modulación de la respuesta alérgica.

\section{REFERENCIAS}

1. National Institutes of Health, National Heart, Lung, and Blood Institute. Global strategy for asthma management and prevention. Revised 2002. Bethesda, MD: NIH; 1995.

2. Stone KD. Atopic diseases of childhood. Curr Opin Pediatr. 2002;14:634-46.

3. Burke W, Fesinmeyer M, Reed K, Hampson L, Carlsten C. Family history as a predictor of asthma risk. Am J Prev Med. 2003;24(2):160-9.

4. Reed SC. Asthma in the United States: burden and current theories. Environ Health Perspect. 2002;110(S4):557-60.

5. Strachan DP. Hay fever, hygiene, and household size. Br Med J. 1989;299:1259-60.

6. Illi S, Von Mutius E, Lau S, Bergmann R, Niggemann B, Sommerfeld C, et al. Early childhood infectious diseases and the development of asthma up to school age: a birth cohort study. Br Med J. 2001;322(7283):390-5.

7. Bresciani M, Parisi C, Manghi G, Bonini S. The hygiene hypothesis: does it function worldwide? Curr Opin Allergy Clin Immunol. 2005;5:147-51.

8. Liu AH, Murphy JR. Hygiene hypothesis: fact or fiction? J Allergy Clin Immunol. 2003;111 (3):471-8.
9. Douwes J, Pearce N. Asthma and the westernisation 'package'. Int J Epidemiol. 2002;31: 1098-102.

10. Braun-Fahrländer C. Environmental exposure to endotoxin and other microbial products and the decreased risk of childhood atopy: evaluating developments since April 2002. Curr Opin Allergy Clin Immunol. 2003;3(5):325-9.

11. Riedler J, Braun-Fahrländer $C$, Eder $W$, Scheuer M, Waser M, Maisch S, et al. Exposure to farming in early life and development of asthma and allergy: a cross-sectional survey. Lancet. 2001;358(9288):1129-33.

12. Kiechl-Kohlendorfer U, Horak E, Mueller W, Strobl R, Haberland C, Fink FM, et al. Neonatal characteristics and risk of atopic asthma in schoolchildren: results from a large prospective birth-cohort study. Acta Paediatr. 2007;96 (11):1606-10.

13. Wickens K, Lane JM, Fitzharris P, Siebers R, Riley G, Douwes J, et al. Farm residence and exposures and the risk of allergic in New Zealand children. Allergy. 2002;57:1171-9.

14. Bråbäck L, Hjern A, Rasmussen F. Trends in asthma, allergic rhinitis and eczema among Swedish conscripts from farming and non- farming environments. A nationwide study over three decades. Clin Exp Allergy. 2004; 34(1):38-43.

15. International Study of Asthma and Allergies in Childhood. Manual. 2nd ed. Auckland, New Zealand: ISAAC; 1993.

16. Vanna AT, Yamada E, Arruda LK, Naspitz CK, Solé D. International Study of Asthma and Allergies in Childhood: validation of the rhinitis symptom questionnaire and prevalence of rhinitis in schoolchildren in São Paulo, Brazil. Pediatr Allergy Immunol. 2001; 12(2):95-101.

17. Hederos CA, Hasselgren M, Hedlin G, Bornehag CG. Comparison of clinically diagnosed asthma with parental assessment of children's asthma in a questionnaire. Pediatr Allergy Immunol. 2007;18(2):135-41.

18. Mata Fernández C, Fernández-Benítez M, Pérez-Miranda M, Guillén Grima F. Validation of the Spanish version of the phase III ISAAC questionnaire on asthma. J Investig Allergol Clin Immunol. 2005;15(3):201-10.

19. Stata Corporation. Stata statistical software: release 8.0. College Station, Texas: Stata Corporation; 2001. 
20. Tatto-Cano I, Sanin-Aguirre L, González V, Ruiz-Velasco S, Romieu I. Prevalencia de asma, rinitis y eczema en escolares de la ciudad de Cuernavaca, México. Salud Publica Mex. 1997;39:497-506.

21. Barraza-Villareal A, Sanin-Aguirre L, TéllezRojo M, Lacasaña-Navarro M, Romieu I. Risk factors for asthma in school children from Ciudad Juárez, Chihuahua. J Asthma. 2003; 40(4):413-23.

22. Barraza-Villareal A, Sanin-Aguirre LH, TéllezRojo MM, Lacasaña-Navarro M, Romieu I. Prevalencia de asma y otras enfermedades alérgicas en niños escolares de Ciudad Juárez, Chihuahua. Salud Publica Mex. 2001;43(5): 433-43.

23. Del Río-Navarro B, Berber A, Blandón V, Ramírez-Aguilar M, Romieu I, RamírezChanona N, et al. Identification of asthma risk factors in Mexico City: an International Study of Asthma and Allergy in Childhood survey. Allergy Asthma Proc. 2006;27(4):325-33.

24. Mallol J, Sole D, Asher I, Clayton T, Stein R, Soto-Quiroz M. Prevalence of asthma symptoms in Latin America: the International Study of Asthma and Allergies in Child- hood (ISAAC). Pediatr Pulmonol. 2000;30: $439-44$.

25. International Study of Asthma and Allergies in Childhood Steering Committee. Worldwide variation in prevalence of symptoms of asthma, allergic rhinoconjunctivitis, and atopic eczema: ISAAC. Lancet. 1998;351 (9111): 1225-32.

26. Gereda JE, Leung DY, Liu AH. Levels of environmental endotoxin and prevalence of atopic disease. J Am Med Assoc. 2000;284 (13): 1652-3.

27. Braun-Fahrländer C, Riedeler J, Herz U, Eder W, Waser M, Grize L, et al. Environmental exposure to endotoxin and its relation to asthma in school-age children. N Engl J Med. 2002;347(12):869-77.

28. Lauener RP, Birchler T, Adamski J, BraunFahrländer C, Bufe A, Herz U, et al. Expression of CD14 and toll-like receptor 2 in farmers' and non- farmers' children. Lancet. 2002; 360(9331):465-6.

29. Prescott SL, Macaubas C, Smallacombe T, Holt BJ, Sly P, Holt PG. Development of allergen-specific T-cell memory in atopic and normal children. Lancet. 1999;353(9148):196-200.
30. Bach JF. The effect of infections on susceptibility to autoimmune and allergic diseases. $\mathrm{N}$ Engl J Med. 2000;347(12):911-20.

31. Stene LC, Nafstad P. Relation between occurrences of type 1 diabetes and asthma. Lancet. 2001;357:607-8.

32. Kero J, Gissier $\mathrm{M}$, Hemminki $\mathrm{E}$, Isolauri $\mathrm{E}$. Could Th1 and Th2 diseases coexist? Evaluation of asthma incidence in children with celiac disease, type 1 diabetes, or rheumatoid arthritis: a register study. J Allergy Clin Immunol. 2001;108(5):781-3.

33. Remes ST, Castro-Rodríguez JA, Holberg CJ Martínez FD, Wright AL. Dog exposure in infancy decreases the subsequent risk of frequent wheeze but not of atopy. J Allergy Clin Immunol. 2001;108(4):509-15.

34. Szklo M, Nieto FJ. Epidemiology: beyond the basics. Geithersburg, MD: Aspen Publisher; 2000.

Manuscrito recibido el 13 de febrero de 2008. Aceptado para publicación, tras revisión, el 17 de septiembre de 2008.

ABSTRACT Objectives. To assess the prevalence of allergic rhinitis symptoms among schoolchildren in Mexicali, Baja California, Mexico, and determine what impact a rural environment might have on this condition.

The effects of a Methods. A population-based, cross-sectional study using a standardized questionnaire administered to a random sample of 2087 schoolchildren 6-7 years of age (1 078 girls and 1009 boys) living in the city of Mexicali, Baja California, Mexico. The study analyzed associations between exposure variables and personal and family health history, and two dependent variables-nasal symptoms and nasal and ocular symptoms, in the absence of a cold or flu-using odd ratios (OR) and 95\% Confidence Intervals (95\%CI). The study took place in February-July 2004 and followed the methodology of the International Study of Asthma and Allergies in Childhood (ISAAC).

Results. The general prevalence of nasal symptoms and nasal and ocular symptoms was $25.0 \%$ and $10.5 \%$, respectively. The boys and girls who had ever lived on a farm or in a rural area had a lower probability, adjusted for confounding variables, of presenting nasal symptoms (OR $=0.43 ; 95 \% \mathrm{CI}$ : $0.24-0.77$ ), or nasal and ocular symptoms (OR $=0.39 ;$ 95\% CI: $0.16-0.93)$.

Conclusions. In the study population, exposure to a rural environment in early childhood decreased the risk of developing allergic rhinitis, regardless of a family history of asthma.

Key words Asthma; rhinitis, allergic, seasonal; rhinitis, allergic, perennial; child; environment and public health; Mexico. 